\title{
Internal quantum efficiency improvement of InGaN/GaN multiple quantum well green light-emitting diodes
}

\author{
Q. ZHOU, M. XU*, and H. WANG \\ Engineering Research Centre for Optoelectronics of Guangdong Province, School of Physics and \\ Optoelectronics, South China University of Technology, Guangzhou, Guangdong 510640, China
}

\begin{abstract}
In recent years, GaN-based light-emitting diode (LED) has been widely used in various applications, such as RGB lighting system, full-colour display and visible-light communication. However, the internal quantum efficiency (IQE) of green LEDs is significantly lower than that of other visible spectrum LED. This phenomenon is called "green gap". This paper briefly describes the physical mechanism of the low IQE for InGaN/GaN multiple quantum well (MQW) green LED at first. The IQE of green LED is limited by the defects and the internal electric field in MQW. Subsequently, we discuss the recent progress in improving the IQE of green LED in detail. These strategies can be divided into two categories. Some of these methods were proposed to enhance crystal quality of InGaN/GaN MQW with high In composition and low density of defects by modifying the growth conditions. Other methods focused on increasing electron-hole wave function overlap by eliminating the polarization effect.
\end{abstract}

Keywords: green LED, internal quantum efficiency, crystal quality, wave function overlap.

\section{Introduction}

In the last decade, GaN-based light-emitting diode (LED) has attracted considerable attention and was seen as a promising replacement of conventional light sources. The spectrum of GaN-based LEDs can be varied from ultraviolet emission to red emission, due to their large and tunable band gaps. As a result, LED was widely used in various applications, such as RGB lighting system, full-colour display and visible-light communication. Compared to currently "phosphor-converted" white LED, RGB lighting system avoids the absorption and emission losses associated with phosphor. Besides, it has better colour rendering and fast response rate. Therefore, RGB white LEDs are the best lighting source in a high quality illumination field.

RGB white LED consists of three kinds of LEDs emitting red, green and blue colours. More than $60 \%$ of internal quantum efficiency (IQE) has been achieved for blue LED [1]. On the other hand, AlGaInP-based LED has been well developed and showed impressive performance in red region, but lost efficiency in shorter wavelengths [2]. The IQE of green LEDs is significantly lower than that of a shorter wavelength InAlGaN-based blue and a longer wavelength InAlGaP-based red LEDs [3]. The lag of the green LED efficiency is called "Green Gap", as shown in Fig. 1.

The emission mechanism in green InGaN/GaN multiple quantum well (MQW) has been studied extensively.

\footnotetext{
*e-mail: phmshxu@ @cut.edu.cn
}

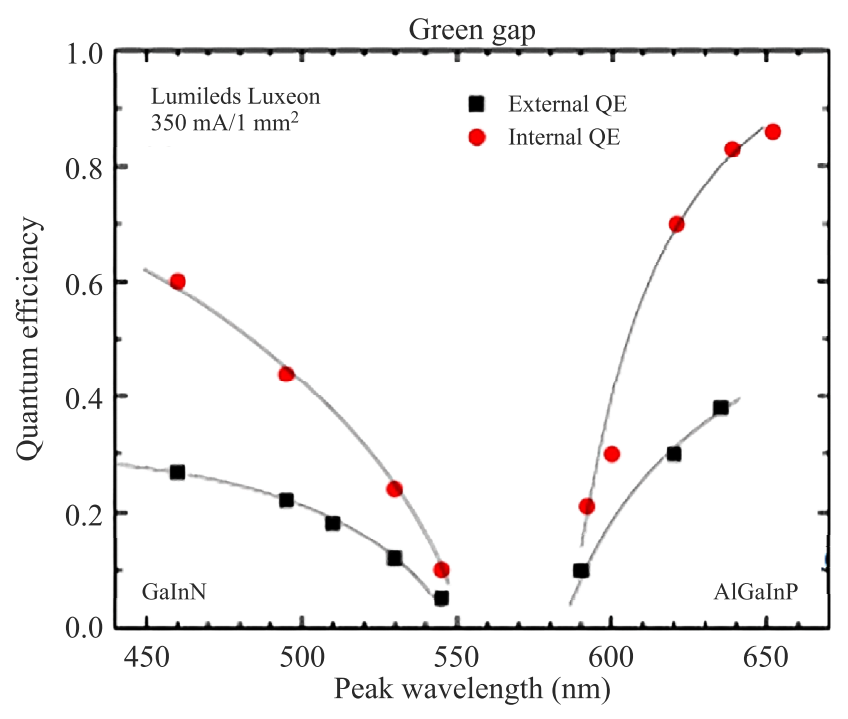

Fig. 1. IQE of an InGaN LED varies with wavelength (reprint from [4]).

Many approaches have been reported recently to overcome the "green gap" for applications of LED. In this paper, we first discussed the mechanisms which are responsible for the low IQE of green InGaN/GaN LED. Then, we reviewed the recent progress in enhancing the IQE of InGaN/GaN-based green LED, including improvement of crystal quality and increase of electron-hole wave function overlap. 


\section{Mechanisms of low IQE for a GaN based green LED}

The InGaN wells' indium composition requires a high indium composition to achieve a long wavelength green LED. The relatively low quantum efficiency of $\mathrm{InGaN} / \mathrm{GaN}$ green MQW with the high indium composition can be attributed to several reasons.

First, it's difficult to grow high quality InGaN/GaN MQWs with a high indium composition. The strain caused by lattice mismatch between $\mathrm{InGaN}$ wells and $\mathrm{GaN}$ barriers may increase with the raise of indium composition in the QWs. Indium clustering induced by a misfit strain is observed when the well thickness is smaller than the critical thickness [5]. However, if the well is thicker than the critical thickness, the misfit strain may cause the misfit strain-induced defects such as point defects [6], impurities [7], V-defects $[8,9]$. These high-density strain-induced defects may act as nonradiative recombination centres in the InGaN/ GaN MQWs and, hence decrease the IQE of the green LED. Additionally, the increase of indium composition also leads to the chemical inhomogeneity of the InGaN well layers due to the poor miscibility between InN and GaN. Phase segregation causes difficulties in achieving homogeneous InGaN alloys, and even a degradation of the InGaN/GaN MQW active region [10].

Secondly, there is an internal electric field in InGaN/ GaN multiple quantum wells of LED grown on the c-plane sapphire. The electrostatic field leads to the spatial separation of electron-hole wave functions and consequently reduces the radiative recombination rates [11]. Because the piezoelectric polarization depends on strain, the reduction in recombination rate is larger for longer emission wavelengths' green LED [2].

\section{Approaches to enhance the efficiency of green LED}

Many efforts have been expanded to overcome the challenges mentioned before. Recently, several approaches have been reported to effectively improve IQE of InGaN/GaN MQW green LED. These strategies can be divided into two categories. The first kind of methods is focused on enhancement of radiative recombination by improving crystal quality of MQW. These methods suppressed the indium segregation and reduce the density of defect by optimizing the growth condition of MQW and employing some new structure. Another kind of means increased the recombination rate by raising the overlap of wave function of holes and electrons, including polarization restraining and energy band engineering design.

\subsection{Improvement of crystal quality}

Normally, the optimal growth temperature of InGaN well layers is substantially lower than that of $\mathrm{GaN}$ barrier layers due to low miscibility of InN in GaN [12]. The conventional method is to grow the whole MQW active region at a constant low temperature since it is difficult to change the growth temperature rapidly and accurately. Consequently, the crystal quality of $\mathrm{GaN}$ barriers grown at low temperature is too poor to get good optoelectronic properties, which is more severe in green LED with high indium component. S.J. Chan et al. improved the crystal quality and light output power of InGaN/GaN MQW green LED by growing a GaN barrier at high temperature $[13,14]$. During the growth of InGaN/GaN MQW, they first ramped down the temperature and waited till it stabilized to grow an InGaN well layer. After that, temperature was ramped up to a higher value and waited till it stabilized to grow a GaN barrier layer. The detail of the temperature ramping process is shown in Fig. 2(a). Figure 2(b) shows the light output power as a function of injection current. The 20-mA output power of nitride-based green LEDs with high temperature GaN barriers increased by $65 \%$ compared to that of the conventional InGaN/GaN green LEDs.

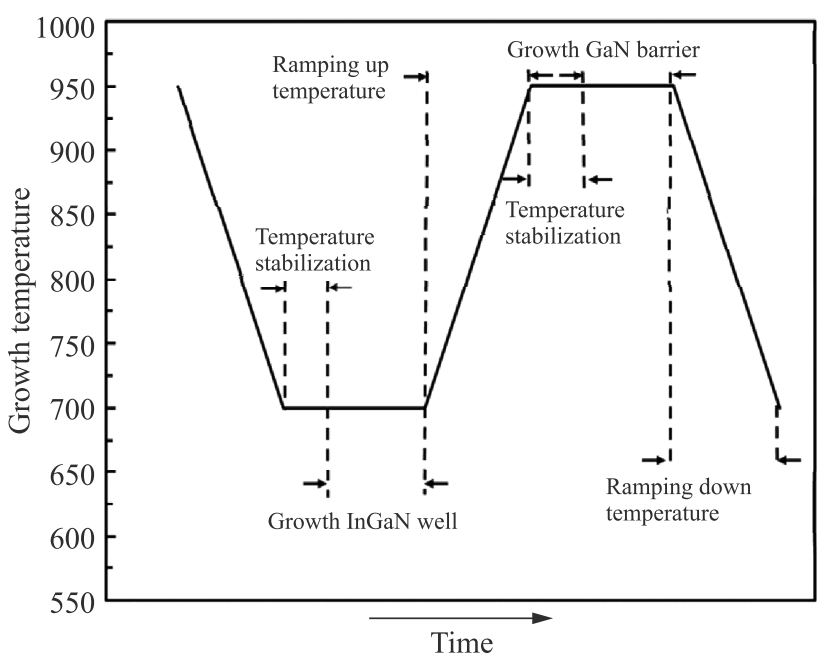

(a)

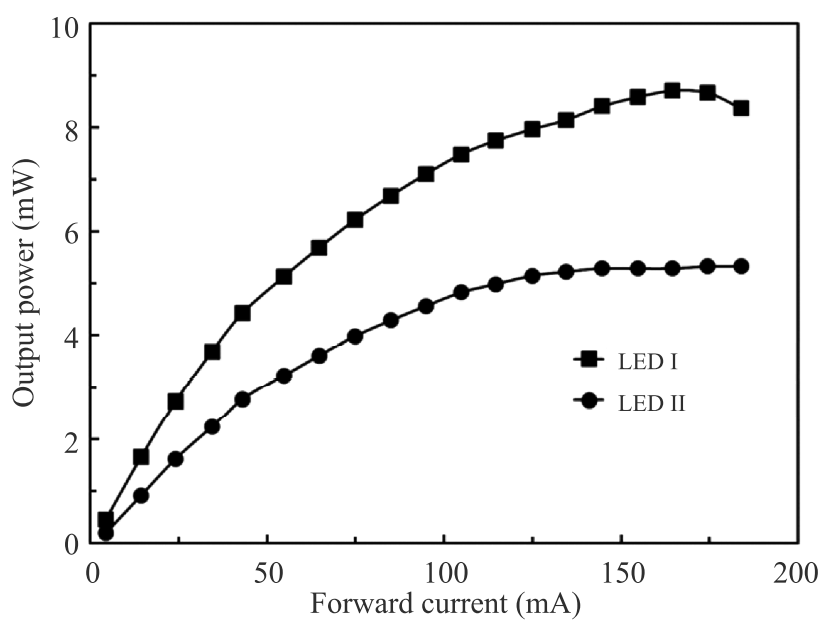

(b)

Fig. 2. (a) Time sequence of the temperature ramping process during the growth of InGaN/GaN MQW active region. (b) Output power of LEDI $b$ (with a temperature ramping MQW) and LEDII (with a constant MQW) (reprinted from Ref. 14). 
However, the high temperature growth GaN barrier may possibly damage the InGaN well layer. During the temperature ramping and barrier growth process, the well suffers from a higher temperature which leads to indium re-evaporation and segregation at the interface of QWs. This thermal degradation of MQW reduces the radiative recombination rate. Jin-Woo Ju et al. used a thin $\mathrm{GaN}$, as well as a protection layer to prevent thermal damage to the fragile well [15]. After the growth of an InGaN well, a 1 nm-thick GaN well-protection-layer was subsequently coated at the same temperature.

Michael Moseley's study revealed that indium surface segregation was more associated with the quantity of indium adsorbed on the surface, rather than growth in the In-rich regime in general [16]. He proposed a method for controlling indium surface segregation by shuttering Group-III metal fluxes at a fixed modulation via metal-modulated epitaxy (MME). The periodic closing of the metal effusion cells allows for complete consumption of excess metal on the surface, preventing droplets from persisting throughout the growth.

Hung-Cheng Lin et al. demonstrated the advantages of using indium treatment in the growth of InGaN/GaN MQW [17]. The indium treatment is to put in an extra step in which only TMIn and $\mathrm{NH}_{3}$ got to flow into the reactor at each InGaN-to-GaN interface during the growth of MQW. As a result, there is a smooth interface and low V-shape defect density in InGaN/GaN MQW, as shown in Figs. 3(a) and 3(b). Figure 3(c) shows the output power of LED with and without indium treatment. The light output power of the TMIn treated LED increases by $43 \%$ compared with that of the untreated LED.

Yufeng Li et al. reported that the GaN epi-layer grown on the patterned sapphire substrate showed a $44 \%$ lower threading dislocation density than that grown on planar substrate [18]. A GaN-based green LED grown on s patterned c-plane sapphire therefore had a doubling of the internal quantum efficiency. Figures 4(a) and 4(b) show cross-section TEM images of $\mathrm{GaN}$ films grown on planar and nano-patterned sapphire. Figures 4(c) and 4(d) show the dramatically enhancement of IQE and EQE nano-patterned substrate LED.

Y. Yang et al. improved the crystallization quality and optoelectronic properties of $\mathrm{InGaN} / \mathrm{GaN} \mathrm{MQW}$ of green LED by growing the structures on freestanding GaN substrates [19]. The density of microstructural defects in the LED on GaN was substantially reduced. As a result, the IQE of green LED on GaN was $71 \%$ higher than similar LED grown on sapphire.

The growth conditions of the MQW play important role in the crystallization qualities and light properties of the green LED. At the same time, the high-temperature post-growth of the p-GaN hole transport layer causes thermal damage to the InGaN/GaN MQW and decreases the emission efficiencies of the InGaN/GaN MQWs LED. The thermal damage of the InGaN/GaN MQW, which is caused by high growth temperature of $\mathrm{p}-\mathrm{GaN}$, is attributed to the in-

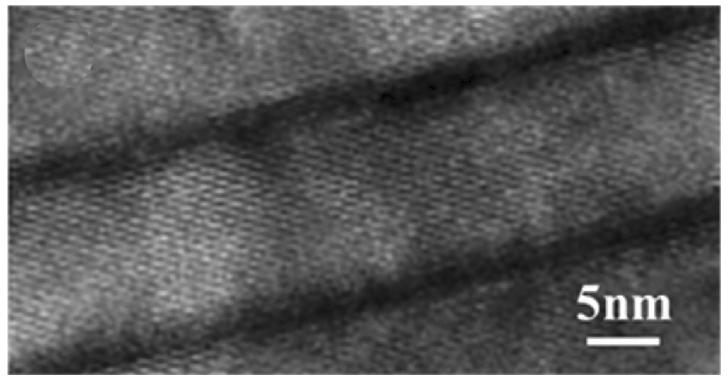

(a)

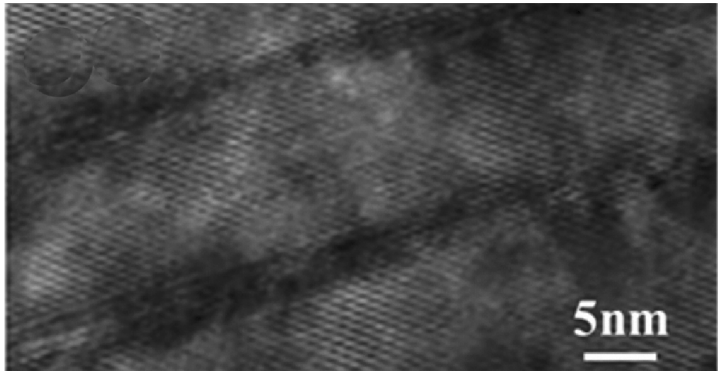

(b)

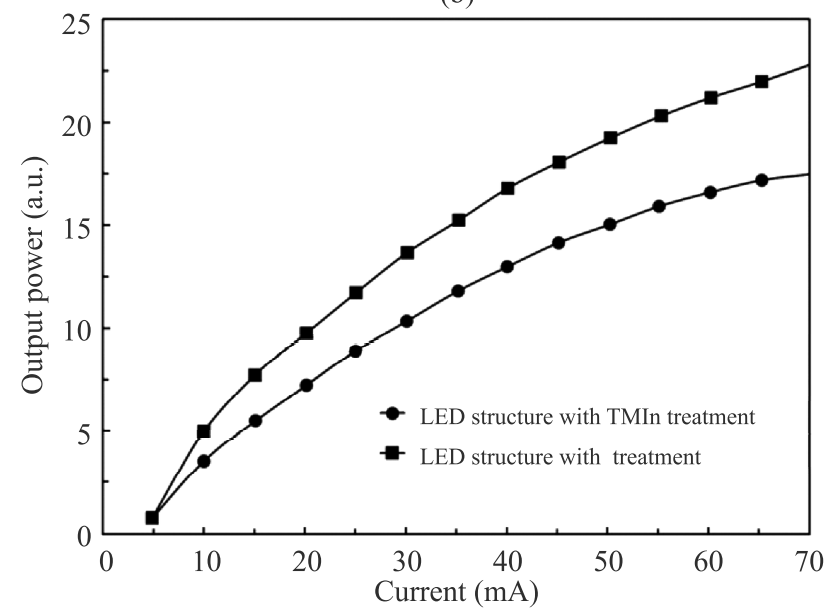

(c)

Fig. 3. HRTEM images of the LEDs grown with (a) and without (b) indium treatment, and (c) light output-current characteristics (reprinted from Ref. 17).

dium diffusion into GaN barrier layers from InGaN well layers [20]. It's promising to reduce the growth temperature of a $\mathrm{p}-\mathrm{GaN}$ layer for better optical and structural properties of InGaN/GaN MQW [21]. The quality of InGaN/GaN MQW green LED could also be improved by "active-region-friendly" p-InGaN layers grown at low temperature [22-24]. Figures 5(a)-5(d) show that structural of MQWs with a $\mathrm{p}-\mathrm{GaN}$ layer grown at different temperature. As a result, the internal quantum efficiency was enhanced due to a better interface quality of the InGaN/GaN MQW [25].

In addition, the interface treatment with $\mathrm{a}_{2}$ flow can suppress the In surface segregation in the interface of QWs. At every stage of interface formation between InGaN well and $\mathrm{GaN}$ barrier, growth is interrupted for an interval and at the same time $\mathrm{H}_{2}$ is introduced into the reactor [26]. The indium segregation effect, which is formed in the InGaN/ GaN MQW and attributed to the subsequent high tempera- 


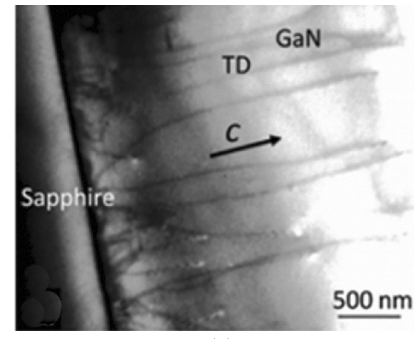

(a)

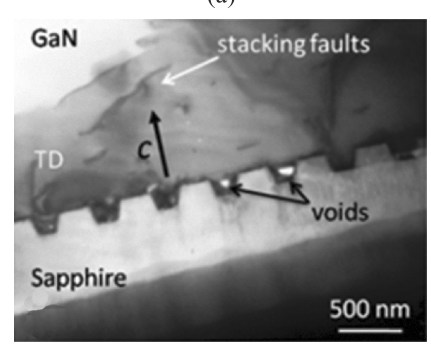

(b)

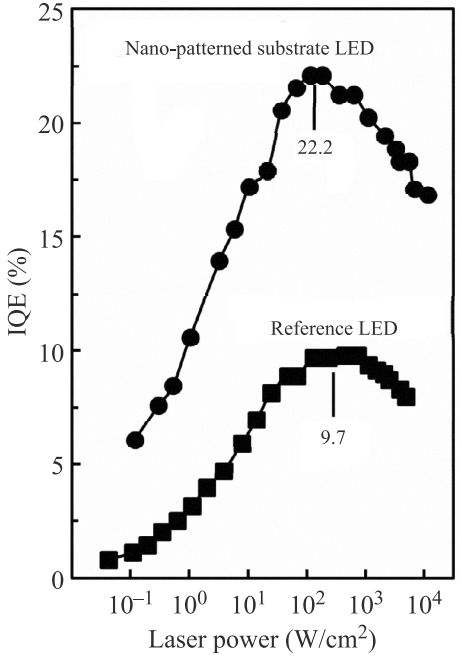

(c)

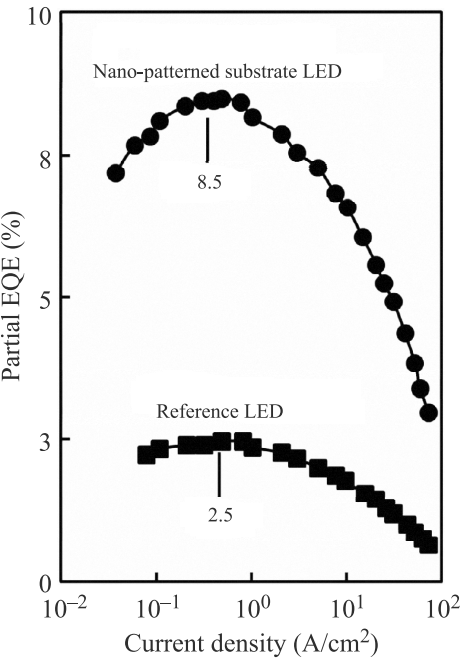

(d)

Fig. 4. (Colour online) Cross-section TEM of the epitaxial GaN/sapphire interface on (a) planar and (b) nano-patterned sapphire. (c) IQE as a function of excitation power density. (d) EQE as a function of current density for the nano-patterned substrate and the reference LED (reprinted from Ref. 18).

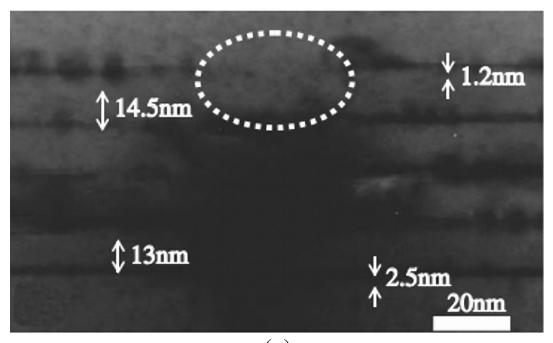

(a)

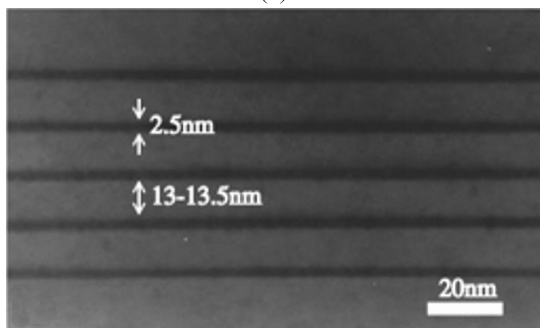

(b)

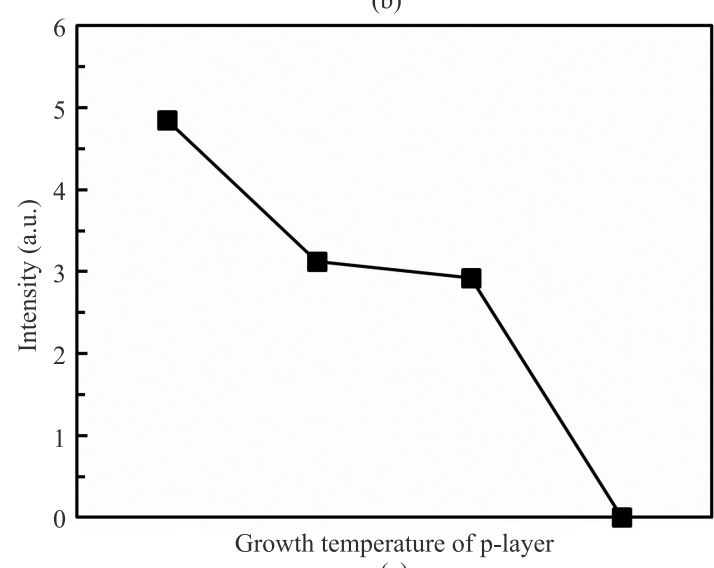

(c)

Fig. 5. Cross-sectional HRTEM images of MQWs with p-GaN epilayers grown at (a) $1050^{\circ} \mathrm{C}$ and (b) $900^{\circ} \mathrm{C}$, and the EDS indium composition profile across (reprinted from Ref. 20), (c) room-temperature PL wavelength and intensity of LED with different p-layer growth temperature (reprinted from Ref. 21). ture growth process of a p-type layer, can be effectively eliminated by introducing $\mathrm{H}_{2}$ gas during the growth interruption period in the growth of MQW [26,27]. As shown in Fig. 6, the photoluminescence intensity of LED drastically increases with raising the $\mathrm{H}_{2}$ flow rate during the growth interruption.

\subsection{Wave functions overlap improvement of electrons and holes}

Polarization electric field directly causes the wave functions separation of electrons and holes. The nonpolar and semipolar InGaN/GaN MQW could efficiently reduce the electrostatic field intensity and increase the quantum efficiency of InGaN QW [28,29].

The r-plane sapphire and a-plane $\mathrm{SiC}$ were used for a nonpolar a-oriented nonpalor $\mathrm{GaN}$ growth [30,31]. Subsequently, a-plane GaN templates [32,34] and m-plane InGaN films on m-plane $6 \mathrm{H}-\mathrm{SiC}$ were reported [35]. Semipolar (10-13) InGaN/GaN green LEDs were also fabricated on an m-plane sapphire [36]. Taeil Jung et al. demonstrated a semipolar green InGaN/GaN MQW fabricated on low cost c-plane sapphire substrates and achieved 30\% higher internal quantum efficiency than a conventional c-plane MQW [37]. In addition, bulk-GaN substrates with arbitrary orientations are attractive to grow a nonpolar and semipolar green LED such as (11-22) orientation GaN [38,39], (20-21) semipolar $\mathrm{GaN}$ [40,41], and a-plane GaN [42].

In the study of Ting-Wei Yeh et al, uniform GaN nanorod arrays were grown vertically by selective area growth on c-plane substrates. These nanorods presented six nonpolar $\{11-00\}$ facets, which served as growth surfaces for semipoalr InGaN/GaN MQW [43], as shown in Figs. 7(a) and 7(b). The absence of polar plane orientation leads to elimination or significant reduction of polarization effects. 


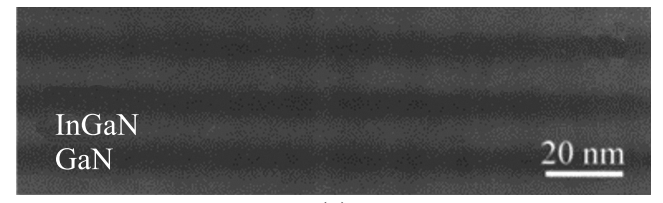

(a)

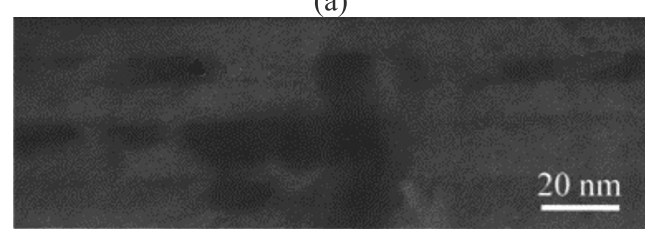

(b)

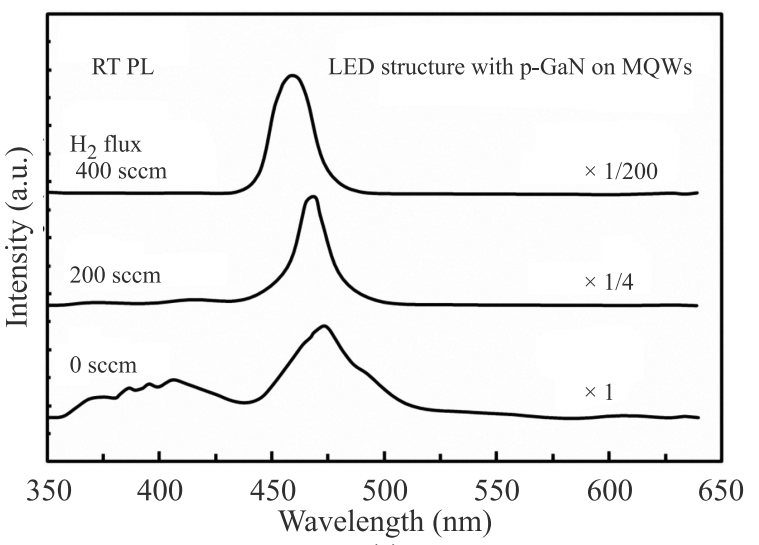

(c)

Fig. 6. Cross sectional images of $\operatorname{InGaN}(6 \mathrm{~nm}) / \mathrm{GaN}(8 \mathrm{~nm}) \mathrm{MQWs}$ grown (a) with $400 \mathrm{sccm} \mathrm{H}_{2}$ and (b) without $\mathrm{H}_{2}$ during the interruption time of 0.5 min., (c) the effect of $\mathrm{H}_{2}$ introduction on the RT PL of a blue LED structure. The $\mathrm{H}_{2}$ was introduced during the interruption time of $0.5 \mathrm{~min}$. (reprinted from Ref. 27).

Relieved QCSE was hence achieved with an improved overlap of electron-hole wave functions for MQW. Consequently, the probability of dipole transition in the semipolar and nonpolar QWs was enhanced for more efficient radiative recombination [44].

Strain is directly responsible for the piezoelectric polarization. Reduction of strain can eliminate the piezoelectric field and improve the overlap of electron-hole wave function. There are some novel methods proposed recently to relax the strain.
One effective method to reduce strain is a strain-compensated growth technique which means to grow MQW with a novel structure to relax strain. D.M. Van Den Broeck et al. reported the growth of $\operatorname{In}_{\mathrm{x}} \mathrm{Ga}_{1-\mathrm{x}} \mathrm{N} / \mathrm{GaN}$ "strainbalanced" multiple quantum wells (SBMQWs) grown on thick $\mathrm{In}_{\mathrm{y}} \mathrm{Ga}_{1-\mathrm{y}} \mathrm{N}$ templates for $\mathrm{x}>\mathrm{y}$ [45]. The SBMQW has been a lattice matched to the thick $\operatorname{In}_{\mathrm{y}} \mathrm{Ga}_{1-\mathrm{y}} \mathrm{N}$ template. In the SBMQWs, thicknesses and compositions are chosen so that the compressive stress in the wells is balanced by the tensile strain in the barriers, as shown in Fig. 8(a). Due to the reduced compressive stress in the SBMQWs, the resulting piezoelectric electric field will be less than conventional InGaN/GaN MQW grown on GaN. This reduction of electric field will increase the overlap of electron-hole wave functions, thus improving the emission intensity. Figure 8(b) shows the obvious enhancement of emission intensity from a conventional MQW to SBMQW.

R. Arvind Pawan et al. designed a novel high-low indium composition and pre-strained the $\mathrm{InGaN} / \mathrm{GaN}$ MQW structure of green LED [46]. In this MQW the first four QWs have a lower indium fraction in $\operatorname{In}_{\mathrm{x}} \mathrm{Ga}_{1-\mathrm{x}} \mathrm{N}$ to minimize lattice strain and achieve better current density. But the fifth layer has a higher indium fraction to get emission in the green region of the spectrum. Such a design with a gradation in indium composition in the QWs considerably reduces the strains in active region and results in the improvement of luminous intensity and radiative recombination rate.

$\mathrm{AlGaN}$ interlayer between the well and the barrier is another approach to compensate strain in InGaN QWs. Shinji Saito et al. employed AlGaN interlayers to enhance the IQE of LED [47]. The AlGaN interlayer which is between the InGaN QW layer and the upper InGaN barrier layer and has $40 \%$ high aluminium content, act as strain-compensating layers for the high-indium-content well layer. As a result, the overlap integral of electron and hole wave functions is enhanced and the crystal quality is improved effectively. Thus, the IQE is improved by the high-aluminium-content AlGaN interlayers.

Hongping Zhao et al. demonstrated a strain-compensated InGaN/AlGaN QW structure employing thin tensile-strained AlGaN barriers to surround the compressively strained InGaN QWs [48]. In addition, Seoung- Hwa n Park et al. reported a strain-compensated $\mathrm{InGaN} / \mathrm{InGaN} \mathrm{QW}$

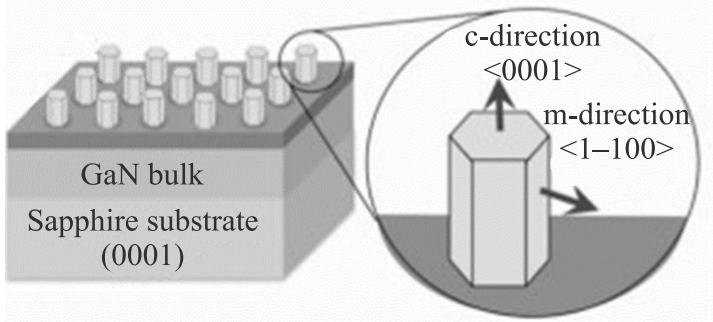

(a)

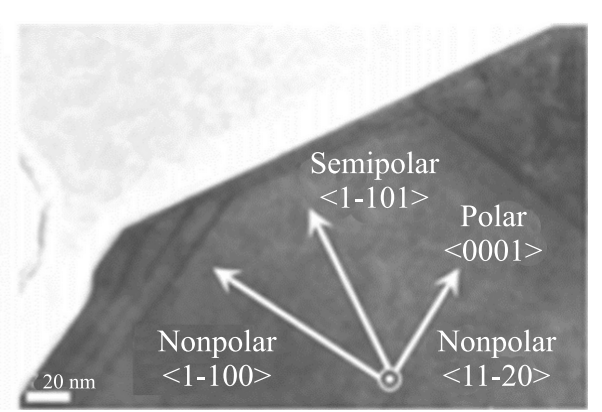

(b)

Fig. 7. (a) Schematic diagram of a GaN nanorod array, (b) InGaN/GaN MQWs are grown on three different planes as indicated in the arrows (reprinted from Ref. 43). 


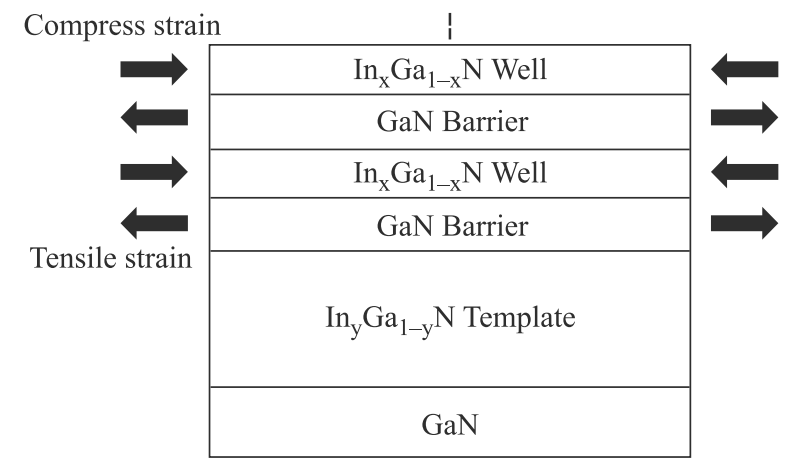

(a)

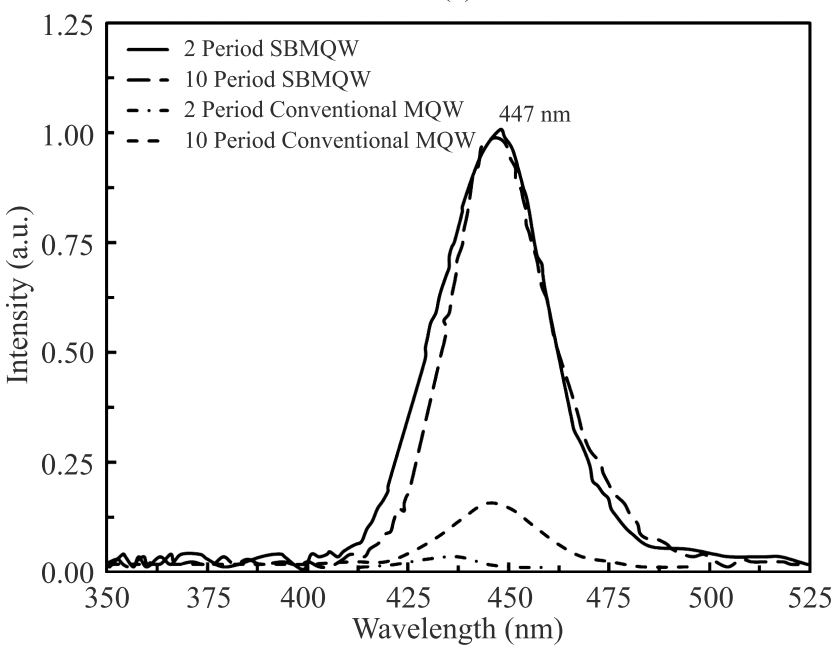

(b)

Fig. 8. Schematics of (a) the proposed $\ln _{\mathrm{x}} \mathrm{Ga}_{1-\mathrm{x}} \mathrm{N} / \mathrm{GaN}$ SBMQW structure grown on a $\operatorname{In}_{\mathrm{y}} \mathrm{Ga}_{1-\mathrm{y}} \mathrm{N}$ template and (b) Normalized PL spectra of 2 and 10 period $\operatorname{In}_{\mathrm{x}} \mathrm{Ga}_{1-\mathrm{x}} \mathrm{N} / \mathrm{GaN}$ SBMQW structures grown on $180 \mathrm{~nm} \mathrm{In}_{\mathrm{y}} \mathrm{Ga}_{1-\mathrm{y}} \mathrm{N}$ templates (reprinted from Ref. 45).

with a tensile-strained InGaN barrier [49]. The radiative efficiency of a strain-compensated QW structure is shown to be much larger than that of a conventional QW structure. This is attributed to the reduction of internal field in the strain-compensated QW structure.

Nanopillar LED is an array of InGaN/GaN MQW rods which is fabricated by inductively coupled plasma etching using a Ni self-assembled nano-size mask, as shown in Fig. 9. The large surface-to-volume ratio of nanopillars provides a way for strain relaxation of $\mathrm{InGaN} / \mathrm{GaN}$ MQW [51]. It means that the piezoelectric field induced by strain reduces and the wave function overlap between electrons and holes increases. In the meantime, the density of defect in $\mathrm{InGaN} / \mathrm{GaN} \mathrm{MQW}$ is significantly reduced. As a result, the radiative recombination rate is enhanced and, thus, the IQE of the LED device is increased. Jin-Woo Ju et al. reported that the PL intensity of the 515 and $543 \mathrm{~nm}$ green nanopillar LED sample had 2.5 times and 7 times improvement respectively, compared to a conventional structure LED [50].

Another way to fabricate a nanopillar LED is a direct growth by metal organic-hydride vapour phase epitaxy. In

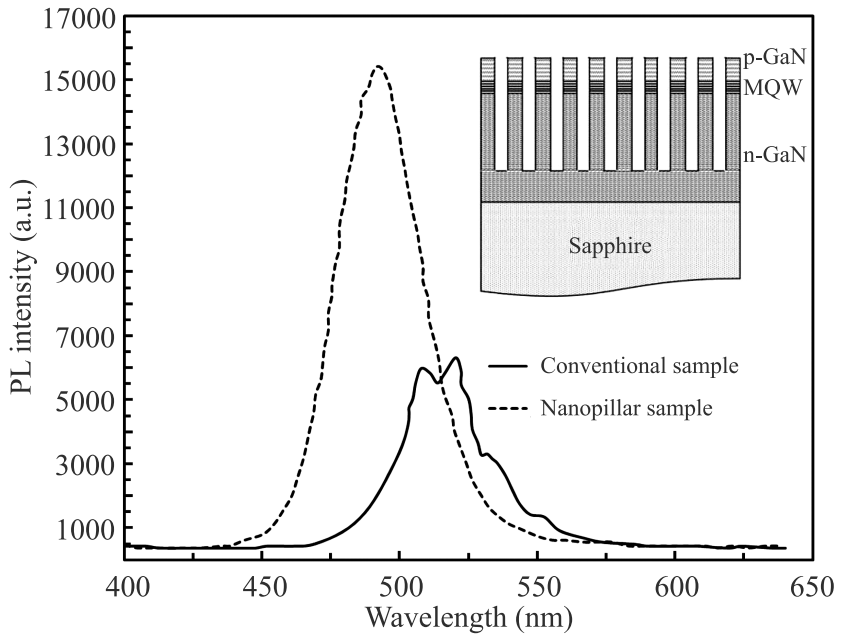

Fig. 9. PL spectra of conventional LED and nanopillar LED. The inset is a schematic diagram of nanopillar LED (reprinted from Ref. 50).

the process of fabrication given by Hwa-Mok Kim et al, nanometer-sized seeds are grown firstly followed by growth of nanopillars on the seeds. Then nanopillar arrays were buried in spin-on glass for the isolation of individual nanopillars, and for the purpose of bringing the p-type NRs into contact with the p-type electrodes [52]. The IQE of a nanopillar LED is significantly increased due to the large sidewall surface area and low dislocation density.

The overlap of electron-hole wave functions can also be increased by the design of staggered InGaN QW. Hongping Zhao et al. enhanced the IQE of green LED by using of the staggered InGaN QW with step-function like In content in the well layer [53-56]. Figure 10 shows the schematic of staggered InGaN QW and L-I curve of green LED with a staggered InGaN QW. The staggered InGaN QW achieves a better overlap of electron-hole wave function, leading to an increase in the radiative recombination rate of the green LEDs.

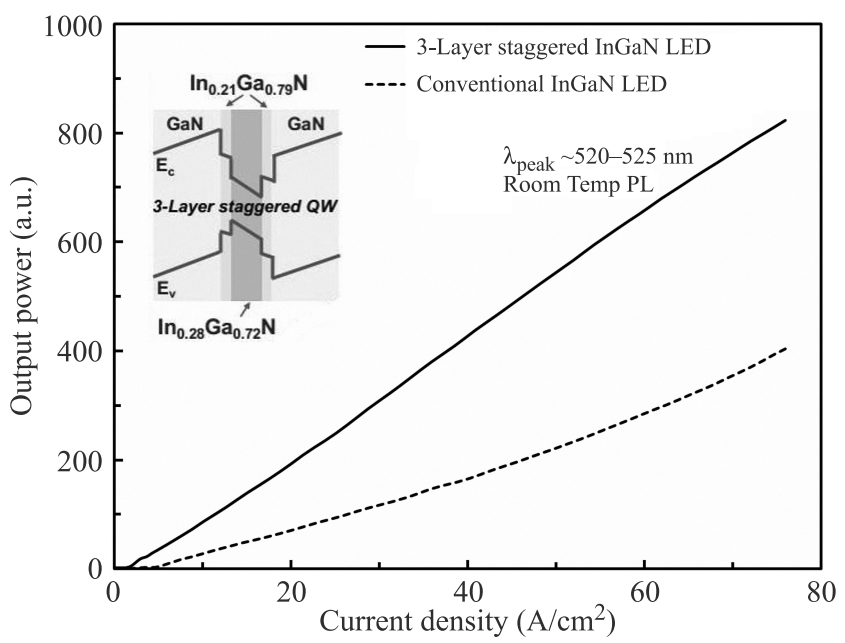

Fig. 10. L-I curve of conventional InGaN QW and three-layer staggered InGaN QW LED. The inset is schematic of the 3-layer staggered InGaN QW structures (reprinted from Ref. 55). 
Moreover, an effective strategy to enhance the recombination efficiency of $\mathrm{InGaN} / \mathrm{GaN}$ based green LED is to form high-density ultra-small In-rich quantum dots (QD) by spontaneous spinodal decomposition in the InGaN active layers. Ultra-small InGaN QDs with uniform size and high density can be self-formed by controlling the $\mathrm{NH}_{3}$ flow rate during the growth of InGaN active layers. Because of the higher In composition in In-rich QDs, the deeper potential levels can attain better capability for carrier confinement and inhibit them from escaping toward surrounding non-radiative recombination centres, such as dislocations and point defects. As a result, InGaN QDs can function as efficient luminescence sources, especially for the longer wavelength spectral region with a high In content, and have exhibited an $18 \%$ enhancement in IQE [57].

\section{Conclusions}

In conclusion, we have discussed the mechanism limited the internal quantum efficiency of InGaN/GaN MQW green LED and recent approaches to enhance the IQE. To achieve green emission, high In composition in InGaN QW is required. Hence, a large lattice mismatch between InGaN wells and GaN barriers occurs as the In composition increases. This effect leads strain-induced defects and strong polarization field. The defects act as nonradiative recombination centres and polarization separates the electron-hole wave function. Consequently, the radiative recombination efficiency of InGaN/GaN MQW green LED is lower than that of blue LED which is called "green gap". Subsequently, we have put emphasis on the recent efforts made to improve efficiency of green LED. Some of these methods were proposed to grow high quality $\mathrm{InGaN} / \mathrm{GaN}$ MQW with high In composition and low density of defects by modifying the growth conditions. Other methods focused on eliminating the polarization effect in MQW by semipolar and nonpolar LED or novel MQW structures.

\section{Acknowledgements}

This work was supported in part by the National High Technology Research and Development Program of China under Grant 2014AA032609, in part by the Strategic Emerging Industry Special Funds of Guangdong Province (2012A080 302003), in part by the National Natural Science Foundation of China (No. 61504044, 61404050), in part by the Fundamental Research Funds for the Central Universities (No.2015ZM074, No. 2014ZM0036).

\section{References}

1. R. Jae-Hyun, P.D. Yoder, L. Jianping, Z. Lochner, K. Hyunsoo, C. Suk, J.K. Hee, and R.D. Dupuis, "Control of quantum-confined stark effect in InGaN-based quantum wells", IEEE J. Sel. Top. Quant. 15, 1080-1091 (2009).

2. M.H. Crawford, "LEDs for Solid-state lighting: performance challenges and recent advances", IEEE J. Sel. Top. Quant. 15, 1028-1040 (2009).
3. Z. Jun, Z. Xiang-Jing, L. Dan-Wei, Y. Lei, L. Kai, Z. Yuan-Wen, D. Jia-Sheng, W. Xing-Fu, and L. Shu-Ti, "Effect of $\mathrm{Mg}$ doping in $\mathrm{GaN}$ interlayer on the performance of green light-emitting diodes", IEEE Photonic. Tech. L 27, 117-120 (2015).

4. M.R. Krames, O.B. Shchekin, R. Mueller-Mach, G.O. Mueller, L. Zhou, G. Harbers, and M.G. Craford, "Status and future of high-power light-emitting diodes for solid-state lighting”, J. Disp. Technol. 3, 160-175 (2007).

5. Y.S. Lin, K.J. Ma, C. Hsu, S. W. Feng, Y.C. Cheng, C.C. Liao, C.C. Yang, C.C. Chou, C.M. Lee, and J.I. Chyi, "Dependence of composition fluctuation on indium content in InGaN/GaN multiple quantum wells", Appl. Phys. Lett. 77, 2988-2990 (2000).

6. J.S. Yue, O. Brandt, and K.H. Ploog, "Photoluminescence intensity of $\mathrm{GaN}$ films with widely varying dislocation density", J. Mater. Res. 18, 1247-250 (2003).

7. D.D. Koleske, A.E. Wickenden, R.L. Henry, and M.E. Twigg, "Influence of MOVPE growth conditions on carbon and silicon concentrations in GaN", J. Cryst. Growth $\mathbf{2 4 2 ,}$ 55-69 (2002).

8. C.J. Sun, M.Z. Anwar, Q. Chen, J.W. Yang, M.A. Khan, M.S. Shur, A.D. Bykhovski, Z. Liliental-Weber, C. Kisielowski, M. Smith, J.Y. Lin, and H.X. Jiang, "Quantum shift of band-edge stimulated emission in InGaN-GaN multiple quantum well light-emitting diodes (vol 70, p. 2978, 1997)", Appl. Phys. Lett. 98, 2978-2980 (1997).

9. F. Scholz, A. Sohmer, J. Off, V. Syganow, A. Dornen, J.S. Im, A. Hangleiter, and H. Lakner, "In incorporation efficiency and composition fluctuations in MOVPE grown GaInN/GaN hetero structures and quantum wells", Materials Science And Engineering B-Solid State Materials For Advanced Technology 50, 238-244 (1997).

10. M.D. McCluskey, L.T. Romano, B.S. Krusor, N.M. Johnson, T. Suski, and J. Jun, "Interdiffusion of In and Ga in InGaN quantum wells", Appl. Phys. Lett. 73, 1281-1283 (1998).

11. R. Jae-Hyun, P.D. Yoder, L. Jianping, Z. Lochner, K. Hyunsoo, C. Suk, J.K. Hee, and R.D. Dupuis, "Control of quantum-confined stark effect in InGaN-based quantum wells", IEEE J. Sel. Top. Quant. 15, 1080-1091 (2009).

12. H. I-Hsiu and G.B. Stringfellow, "Solid phase immiscibility in GaInN", Appl. Phys. Lett. 69, 2701-2703 (1996).

13. C.H. Liu, Y.K. Su, T.C. Wen, S.J. Chang, and R.W. Chuang, "Nitride-based green light emitting diodes grown by temperature ramping", J. Cryst. Growth. 254, 336-341 (2003).

14. L.W. Wu, S.J. Chang, Y.K. Su, R.W. Chuang, T.C. Wen, C.H. Kuo, W.C. Lai, C.S. Chang, J.M. Tsai, and J.K. Sheu, "Nitride-based green light-emitting diodes with high temperature GaN barrier layers", IEEE T. Electron. Dev. 50, 1766-1770 (2003).

15. J. Ju, H. Kim, L. Jang, J.H. Baek, D. Shin, and I. Lee, "A well protection layer as a novel pathway to increase indium composition: a route towards green emission from a blue $\mathrm{InGaN} /$ GaN multiple quantum well", Nanotechnology 18, 460-464 (2007).

16. M. Moseley, J. Lowder, D. Billingsley, and W.A. Doolittle, "Control of surface adatom kinetics for the growth of high-indium content InGaN throughout the miscibility gap", Appl. Phys. Lett. 97, 191902-191902-3 (2010).

17. L. Hung-Cheng, L. Ruo-Syuan, and C. Jen-Inn, "Enhancing the quantum efficiency of InGaN green light-emitting diodes 
by trimethylindium treatment", Appl. Phys. Lett. 92, 161111-161113 (2008).

18. L. Yufeng, Y. Shi, Z. Mingwei, Z. Liang, H. Wenting, T. Detchprohm, Y. Taniguchi, N. Tamura, S. Tanaka, and C. Wetzel, "Defect-reduced green GaInN/GaN light-emitting diode on nanopatterned sapphire", Appl. Phys. Lett. 98, 151102-151103 (2011).

19. Y. Yang, X.A. Cao, and C.H. Yan, "Rapid efficiency roll-off in high-quality green light-emitting diodes on freestanding GaN substrates", Appl. Phys. Lett. 94, 041117-041117-3 (2009).

20. M. Oh, M. Kwon, I. Park, S. Baek, S. Park, S.H. Lee, and J.J. Jung, "Improvement of green LED by growing $\mathrm{p}-\mathrm{GaN}$ on In0.25GaN/GaN MQWs at low temperature", J. Cryst. Growth. 289, 107112 (2006)

21. W. Lee, J. Limb, J.H. Ryou, D. Yoo, T. Chung, and R.D. Dupuis, "Influence of growth temperature and growth rate of $\mathrm{p}-\mathrm{GaN}$ layers on the characteristics of green light emitting diodes", J. Electron. Mater. 35, 587-591 (2006).

22. W. Lee, J. Limb, J. Ryou, D. Yoo, T. Chung, and R.D. Dupuis, "Effect of thermal annealing induced by p-type layer growth on blue and green LED performance", J. Cryst. Growth. 287, 577-581 (2006).

23. L. Wonseok, L. Jae, R. Jae-Hyun, Y. Dongwon, M.A. Ewing, Y. Korenblit, and R.D. Dupuis, "Nitride-based Green light-emitting diodes with various p-type layers", J. Disp. Technol. 3, 126-132 (2007).

24. J.B. Limb, W. Lee, J.H. Ryou, D. Yoo, and R.D. Dupuis, "Comparison of $\mathrm{GaN}$ and $\operatorname{In}(0.0) 4 \mathrm{Ga}(0.96) \mathrm{N}$ p-layers on the electrical and electroluminescence properties of green light emitting diodes", J. Electron. Mater. 36, 426-430 (2007).

25. J. Ju, J. Zhu, H. Kim, C. Lee, and I. Lee, "Effects of p-GaN growth temperature on a green $\mathrm{InGaN} / \mathrm{GaN}$ multiple quantum well", J. Korean Phys. Soc. 50, 810-813 (2007).

26. W. Liu, S.J. Chua, X.H. Zhang, and J. Zhang, "Effect of high temperature and interface treatments on photoluminescence from InGaN/GaN multiple quantum wells with green light emissions", Appl. Phys. Lett. 83, 914-916 (2003).

27. M. Yong-Tae, K. Dong-Joon, S. Keun-Man, C. Chel-Jong, H. Sang-Heon, S. Tae-Yeon, and P. Seong-Ju, "Effects of thermal and hydrogen treatment on indium segregation in InGaN/GaN multiple quantum wells", J. Appl. Phys. 89, 6514-6518 (2001).

28. S. Qian and H. Jung, "Nonpolar and semipolar GaN heteroepitaxy on sapphire for LED application", Proc. SPIE 7617, 761714-761717 (2010).

29. H. Masui, S. Nakamura, S.P. DenBaars, and U.K. Mishra, "Nonpolar and semipolar iii-nitride light-emitting diodes: achievements and challenges", IEEE T. Electron. Dev. 57, 88-100 (2010).

30. M.D. Craven, S.H. Lim, F. Wu, J.S. Speck, and S.P. DenBaars, "Nonpolar (11(2)over-bar0) a-plane gallium nitride thin films grown on (1(1)over-bar02) r-plane sapphire: Heteroepitaxy and lateral overgrowth", Phys. Status Solidi A. 194, 541-544 (2002).

31. M.D. Craven, A. Chakraborty, B. Imer, F. Wu, S. Keller, U.K. Mishra, J.S. Speck, and S.P. DenBaars, "Structural and electrical characterization of a-plane GaN grown on a-plane SiC," in Phys. Status Solidi C. M. Stutzmann, pp. 21322135, ed. Wiley-Vch, Inc, New York, 2003.

32. C.Q. Chen, J.P. Zhang, J.W. Yang, V. Adivarahan, S. Rai, S. Wu, H.M. Wang, W.H. Sun, M. Su, Z. Gong, E. Kuokstis, M.
Gaevski, and M.A. Khan, "A new selective area lateral epitaxy approach for depositing a-plane $\mathrm{GaN}$ over r-plane sapphire", Japanese J. Appl. Phys. Part 2-Letters 42, L818L820 (2003).

33. B.M. Imer, F. Wu, S.P. DenBaars, and J.S. Speck, "Improved quality (11(2)over-bar-0) a-plane GaN with sidewall lateral epitaxial overgrowth", Appl. Phys. Lett. 88, 061908061908-3 (2006).

34. D. Iida, A. Miura, Y. Okadome, Y. Tsuchiya, T. Kawashima, T. Nagai, M. Iwaya, S. Kamiyama, H. Amano, and I. Akasaki, "One-step lateral growth for reduction in defect density of a-plane GaN on r-sapphire substrate and its application in light emitters", Phys. Status Solidi A. 204, 2005-2009 (2007).

35. M. Iwaya, A. Miura, R. Senda, T. Nagai, T. Kawashima, D. Iida, S. Kamiyama, H. Amano, and I. Akasaki, "Control of stress and crystalline quality in GaInN films used for green emitters", J. Cryst. Growth 310, 4920-4922 (2008).

36. R. Sharma, P.M. Pattison, H. Masui, R.M. Farrell, T.J. Baker, B.A. Haskell, F. Wu, S.P. DenBaars, J.S. Speck, and S. Nakamura, "Demonstration of a semi polar (10(1)over-bar1(3)over-bar) InGaN/GaN green light emitting diode", Appl. Phys. Lett. 87, 231110-231110-3 (2005).

37. T. Jung, T. Jung, L. Lee, L. Lee, P.C. Ku, and P.E. Ku, "Semi-polar green LEDs on c-plane sapphire substrates", IEEE, 571-572, (2008).

38. M. Funato, M. Ueda, Y. Kawakami, Y. Narukawa, T. Kosugi, M. Takahashi, and T. Mukai, "Blue, green, and amber InGaN/GaN light-emitting diodes on semipolar $\{1122\} \mathrm{GaN}$ bulk substrates", Jpn J. Appl. Phys. Part 2-Letters \& Express Letters 45, L659-L662 (2006).

39. H. Sato, A. Tyagi, H. Zhong, N. Fellows, R.B. Chung, M. Saito, K. Fujito, J.S. Speck, S.P. DenBaars, and S. Nakamura, "High power and high efficiency green light emitting diode on free-standing semipolar (11(2)over-bar2) bulk GaN substrate", Phys Status Solidi-R 1, 162-164 (2007).

40. R. B. Chung, Y. Lin, I. Koslow, N. Pfaff, H. Ohta, J. Ha, S.P. DenBaars, and S. Nakamura, "Electroluminescence characterization of (20(2)over-bar1) InGaN/GaN light emitting diodes with various wavelengths", Jpn. J. Appl. Phys. 49, 070203-070203-3 (2010).

41. S. Yamamoto, Y. Zhao, C. Pan, R.B. Chung, K. Fujito, J. Sonoda, S.P. DenBaars, and S. Nakamura, "High-efficiency single-quantum-well green and yellow-green light-emitting diodes on semipolar (20(2)over-bar1) GaN substrates", Appl. Phys. Express 3, 122102-122102-3 (2010).

42. T. Detchprohm, M. Zhu, Y. Li, Y. Xia, C. Wetzel, E.A. Preble, L. Liu, T. Paskova, and D. Hanser, "Green light emitting diodes on a-plane GaN bulk substrates", Appl. Phys. Lett. 92, 241109-241109-3 (2008).

43. T. Yeh, Y. Lin, L.S. Stewart, P.D. Dapkus, R. Sarkissian, J.D. O'Brien, B. Ahn, and S.R. Nutt, "InGaN/GaN multiple quantum wells grown on nonpolar facets of vertical GaN nanorod arrays", Nano. Lett. 12, 3257-3262 (2012).

44. G. You, J. Liu, Z. Jiang, L. Wang, N.A. El-Masry, A.M. Hosalli, S.M. Bedair, and J. Xu, "Enhanced radiative recombination and suppressed Auger process in semipolar and nonpolar InGaN/GaN quantum wells grown over GaN nanowires", Opt. Lett. 39, 1501-1504 (2014).

45. D.M. Van den Broeck, D. Bharrat, A.M. Hosalli, N.A. El-Masry, and S.M. Bedair, "Strain-balanced InGaN/GaN mul- 
tiple quantum wells", Appl. Phys. Lett. 105, $031107-$ 031107-5 (2014)

46. P.R. Arvind, P.R. Arvind, C. Dhanavantri, and C. Dhanavantri, "Optimization of optical behavior of InGaN-GaN MQW green LEDs with a novel high-low profile of Indium composition in the active layer", IEEE, pp. 748-751 (2009).

47. S. Saito, R. Hashimoto, J. Hwang, and S. Nunoue, "InGaN light-emitting diodes on c-face sapphire substrates in green gap spectral range", Appl. Phys. Express 6, 207-211 (2013).

48. H. Zhao, R.A. Arif, Y. Ee, and N. Tansu, "Self-consistent analysis of strain-compensated InGaN-AlGaN quantum wells for lasers and light-emitting diodes", IEEE J Quantum Elect 45, 66-78 (2009).

49. P. Seoung-Hwan, M. Yong-Tae, S.L. Jeong, K.K. Ho, S.P. Joong, and A. Doyeol, "Spontaneous emission rate of green strain-compensated InGaN/InGaN LEDs using InGaN substrate", Phys. Status Solidi A 208, 195-198 (2011).

50. J. Ju, J.H. Baek, S. Lee, D. Jeon, J. Park, J. Choi, L. Jang, and I. Lee, "Fabrication and characterization of high efficiency green nanopillar LED”, 332-335(2013).

51. Z. Ji-Hong, S. Yong-Sheng, L. Su-Ying, Y. Hui, Z. Shu-Ming, S.ô.D.Z. Xian, Z. Jian-Jun, L. Zong-Shun, J. De-Sheng, D. Li-Hong, and W. Hai, "Fabrication and optical characterization of gan-based nanopillar light emitting diodes", Chinese Phys. Lett. 25, 3485-3488 (2008).

52. H.M. Kim, Y.H. Cho, H. Lee, S.I. Kim, S.R. Ryu, D.Y. Kim, T.W. Kang, and K.S. Chung, "High-brightness light emitting diodes using dislocation-free indium gallium nitride/gallium nitride multiquantum-well nanorod arrays", Nano. Lett. 4, 1059-1062 (2004).

53. Z. Hongping, R.A. Arif, and N. Tansu, "Design analysis of staggered InGaN quantum wells light-emitting diodes at 500-540 nm", IEEE J. Sel. Top. Quant. 15, 1104-1114 (2009).

54. H.P. Zhao, G.Y. Liu, X.H. Li, R.A. Arif, G.S. Huang, J.D. Poplawsky, S.T. Penn, V. Dierolf, and N. Tansu, "Design and characteristics of staggered InGaN quantum-well light-emitting diodes in the green spectral regime", IET Optoelectron. 3, 283-295 (2009).

55. Z. Hongping, L. Guangyu, L. Xiao-Hang, G.S. Huang, J.D. Poplawsky, S.T. Penn, V. Dierolf, and N. Tansu, "Growths of staggered InGaN quantum wells light-emitting diodes emitting at 520-525 nm employing graded growth-temperature profile", Appl. Phys. Lett. 95, 61103-61104 (2009).

56. Z. Hongping, L. Guangyu, Z. Jing, J.D. Poplawsky, V. Dierolf, and N. Tansu, "Approaches for high internal quantum efficiency green InGaN light-emitting diodes with large overlap quantum wells", Opt. Express 19, A991- 1007 (2011).

57. L. Cheng-Hsueh, L. Yi-Chang, C. Yen-Hsiang, T. Sheng-Chieh, L. Yen-Lin, L. Yun-Li, and L. Chuan-Pu, "Output power enhancement of InGaN/GaN based green light-emitting diodes with high-density ultra-small In-rich quantum dots", J. Alloy Compd. 555, 250-254 (2013). 